\title{
and Meta-analysis of Randomized Controlled Trials
}

\author{
Qiang Su, MD; Lang Li, MD, PhD; Yangchun Liu, MD
}

Department of Cardiology, The First Affiliated Hospital of Guangxi Medical University, Guangxi

Cardiovascular Institute, Nanning, China

Background: To evaluate the clinical efficacy and safety of intracoronary verapamil injection in the prevention and treatment of coronary no-reflow after percutaneous coronary intervention (PCI).

Hypothesis: Intracoronary verapamil injection may be beneficial in preventing no-reflow/slow-flow after PCI. Methods: We searched PubMed, Embase, and the Cochrane Central Register of Controlled Trials database. Randomized trials comparing the efficacy and safety of intracoronary verapamil infusion vs control in patients with acute coronary syndrome (ACS) were included. Meta-analysis was performed by RevMan 5.0 software (Cochrane Collaboration, Copenhagen, Denmark).

Results: Seven trials involving 539 patients were included in the analysis. Verapamil treatment was significantly more effective in decreasing the incidence of no-reflow (risk ratio [RR]: 0.33; 95\% confidence interval [CI]: 0.23 to 0.50 ) as well as reducing the corrected thrombolysis in myocardial infarction (TIMI) frame count (CTFC) (weighted mean difference: $-11.62 ; 95 \% \mathrm{Cl}:-16.04$ to -7.21 ) and improving the TIMI myocardial perfusion grade (TMPG) (RR: $0.43 ; 95 \% \mathrm{Cl}: 0.29$ to 0.64 ). Verapamil also reduced the 30 -day wall motion index (WMI) compared to the control. Moreover, the procedure reduced the incidence of major adverse cardiac events (MACEs) in ACS patients during hospitalization (RR: $0.37 ; 95 \% \mathrm{Cl}: 0.17$ to 0.80 ) and 2 months after $\mathrm{PCl}$ (RR: $0.56 ; 95 \% \mathrm{Cl}: 0.33$ to 0.95 ). However, administration of verapamil did not provide an additional improvement of left ventricular ejection fraction regardless of the time that had passed post-PCl.

Conclusions: Intracoronary verapamil injection is beneficial in preventing no-reflow/slow-flow, reducing CTFC, improving TMPG, and lowering WMI. It is also likely to reduce the 2-month MACEs in ACS patients post-PCI.

\section{Introduction}

Percutaneous coronary intervention (PCI) is the preferred reperfusion strategy for acute coronary syndrome. Despite optimal evidence-based PCI, myocardial no-reflow can still occur and is associated with a worse in-hospital and longterm prognosis. ${ }^{1}$ The concept of no-reflow refers to a state of myocardial tissue hypoperfusion and microcirculatory dysfunction in the presence of a patent epicardial coronary artery. Reperfusion no-reflow is an independent predictor of short- and long-term adverse cardiac events and mortality following $\mathrm{PCI} .^{2}$ As the process is multifactorial, various therapeutic strategies are required in different situations. Current pharmacological management involves the use

The authors have no funding, financial relationships, or conflicts of interest to disclose. of vasodilators, including nitrates, verapamil, papaverine, adenosine, nicardipine, and sodium nitroprusside, but interestingly, vasoconstrictors such as epinephrine may also have a role. ${ }^{3}$ Verapamil is a calcium channel blocker (CCB) that improves vascular wall stress and increases myocardial perfusion by disrupting the movement of calcium $\left(\mathrm{Ca}^{2+}\right)$ through calcium channels in coronary arteries. ${ }^{4}$ Based on the mechanism of action of verapamil, this CCB agent has been widely used in catheter labs to prevent and treat coronary no-reflow. However, a limited number of clinical studies have reported conflicting results, especially in patients with acute coronary syndrome (ACS). Thus, the value of verapamil in improving coronary no-reflow associated with PCI has not been well established. Therefore, in this study, we conducted a meta-analysis of randomized controlled trials to assess the clinical efficacy and safety of verapamil in patients with ACS. 


\section{Methods}

\section{Inclusion Criteria}

Trials were included if (1) they enrolled patients with a diagnosis of ACS regardless of non-ST-segment elevation ACS or ST-segment elevation myocardial infarction; (2) studies that randomly allocated patients with coronary no-reflow to undergo intracoronary infusion of verapamil or placebo/blank control during the coronary interventional procedure; and (3) they reported information on thrombolysis in myocardial infarction (TIMI) classification, major adverse ca rdiac events (MACEs) (such as all-cause death, target vessel revascularization, recurrent angina or myocardial infarction, and severe heart failure), left ventricular ejection fraction (LVEF), or wall motion index (WMI).

\section{Search Strategy}

Relevant studies were identified through a computerized literature search of PubMed, Embase, and Cochrane databases (Central Register of Controlled Trials and Cochrane Database of Systematic Reviews) through July 2012 using the search terms "slow flow," "no reflow," "percutaneous coronary intervention," "verapamil,” "randomized," and "human." To ensure that no clinical trials were missed, we also conducted an extensive search of a citation database (ISI Web of Science) using cross references from eligible articles. No language restrictions were used.

\section{Data Extraction and Quality Assessment}

Two authors (S.Q. and L.Y.) independently reviewed the data and assessed the eligibility and methodological quality of each eligible trial. Information regarding study and patient characteristics, intervention strategies, and the prespecified clinical outcomes was systematically extracted. Disagreements were discussed between the authors, and if the authors could not reach a consensus, disagreements were resolved by the third author (L.L.). The bias risk of trials was assessed with the components recommended by the Cochrane Collaboration, ${ }^{5}$ including sequence generation of the allocation, allocation concealment, blinding of

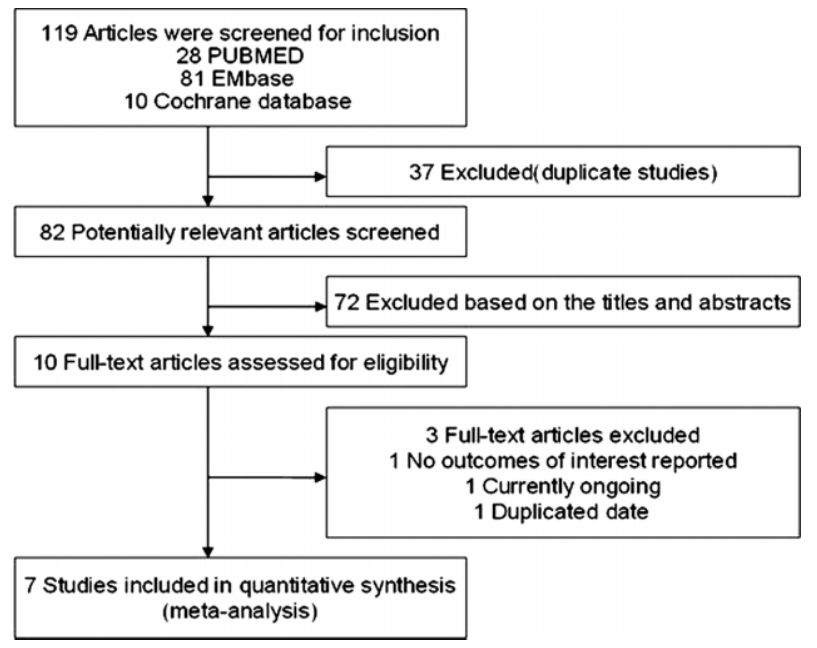

Figure 1. Flow chart of study selection.

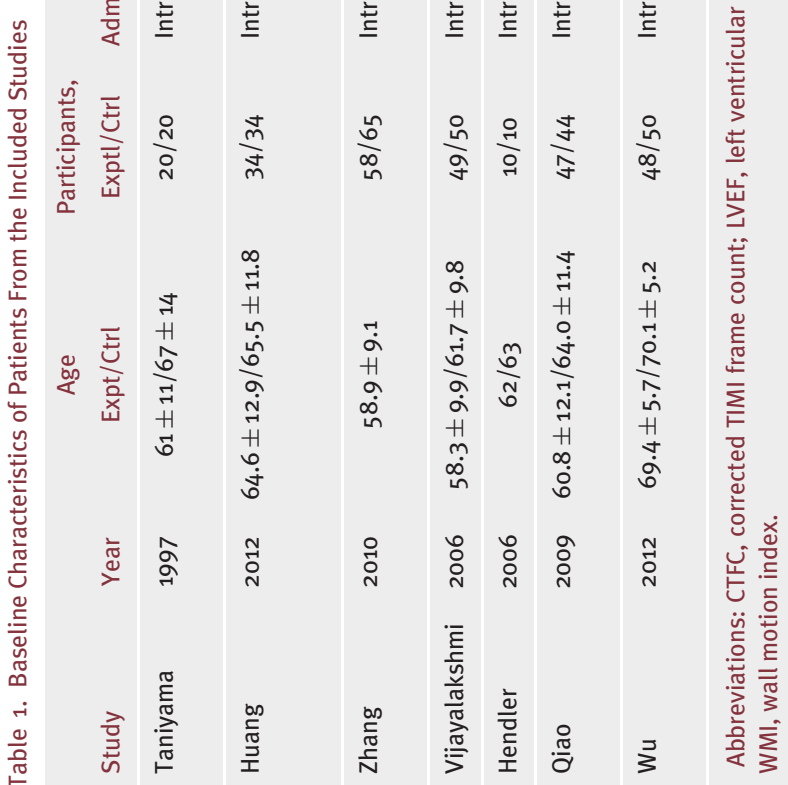


Table 2. Assessment of Methodological Quality of Included Studies

\begin{tabular}{|c|c|c|c|c|c|}
\hline Study & Randomized Method & Allocation Concealment & Blinded & Withdrawals and Lost to Follow-up & Intent-to-Treat Analysis \\
\hline Taniyama 1997 & Randomized unclear & Unclear & Unclear & No & No \\
\hline Huang 2012 & Randomized block design & Unclear & Single blind & No & No \\
\hline Zhang 2010 & Randomized unclear & Unclear & Unclear & No & No \\
\hline Vijayalakshmi 2006 & Randomized unclear & Unclear & Unclear & No & No \\
\hline Hendler 2006 & Randomized unclear & Unclear & Single blind & No & No \\
\hline Qiao 2009 & Randomized number table & Unclear & Double blind & No & No \\
\hline Wu 2012 & Randomized number table & Unclear & Double blind & No & No \\
\hline
\end{tabular}

participants, personnel, and outcome assessors, incomplete outcome data, selective outcome reporting, and other sources of bias.

The primary end point was hospital and 2-month MACE. Secondary end points were TIMI flow grade, corrected TIMI frame count (CTFC), and LVEF in hospital and 1-month follow-up. MACE indicated major adverse cardiac events and was defined as the composite of death, myocardial infarction, and target vessel revascularization.

\section{Data Analyses}

Risk ratio (RR) with 95\% confidence interval (CI) was used to express the pooled effect on discontinuous variables. The summary estimates of continuous variables were presented as weighted mean differences (WMD) with 95\% CI. Heterogeneity was quantified using $I^{2}$ statistic, where $I^{2}>50 \%$ represented between-study inconsistency. Fixed-effects meta-analyses were conducted to pool these outcomes across the included trials when there was no between-study inconsistency. Publication bias was evaluated using a funnel plot. Results were considered statistically significant at $P<0.05$. The pooled analyses were performed with ReviewManager 5.0 software (Cochrane Collaboration, Copenhagen, Denmark).

\section{Results}

\section{Selected Studies and Baseline Characteristics}

Figure 1 shows the flow of the process for identifying potentially eligible trials and reasons for exclusion. From the initial

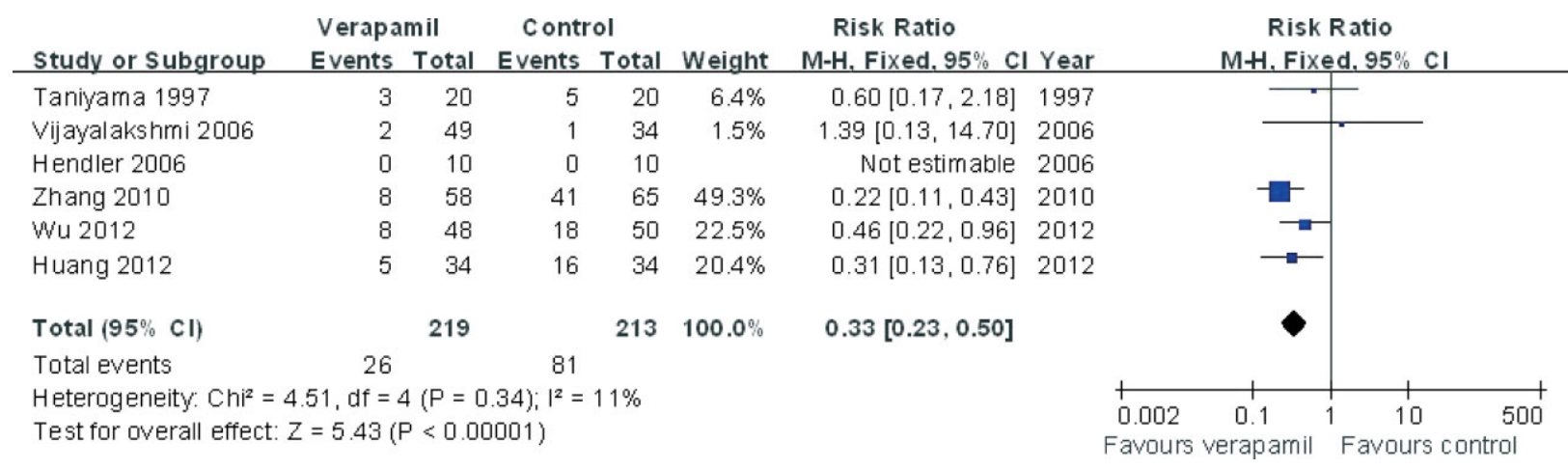

Figure 2. Pooled risk ratio of verapamil therapy vs control for no-flow/slow-flow after percutaneous coronary intervention. Abbreviations: Cl, confidence interval; M-H, Mantel-Haenszel.

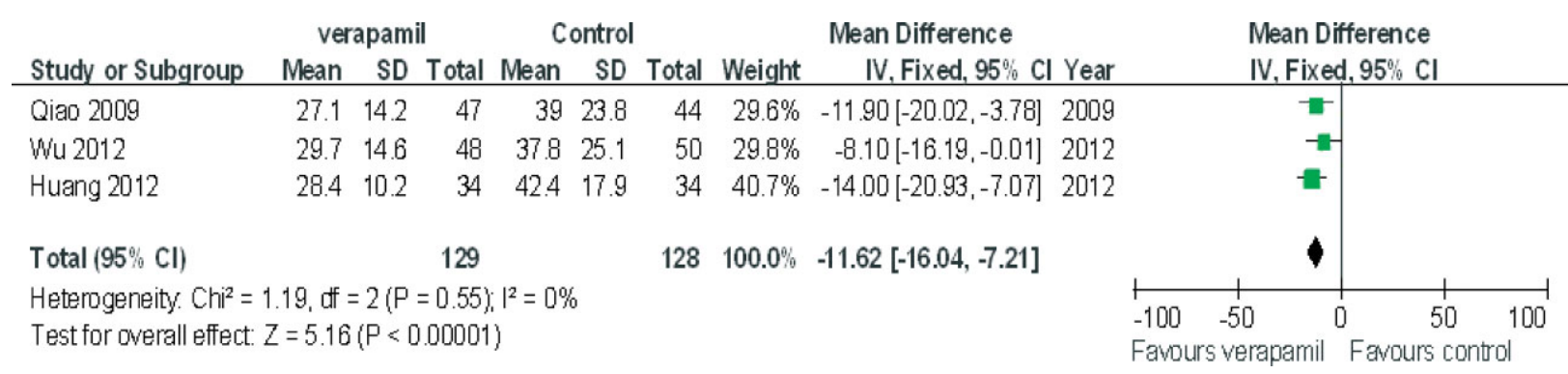

Figure 3. Pooled mean difference of verapamil therapy vs control for correct thrombolysis in myocardial infarction frame count after percutaneous coronary intervention. Abbreviations: Cl, confidence intervals; IV, inverse variance; SD, standard deviation. 


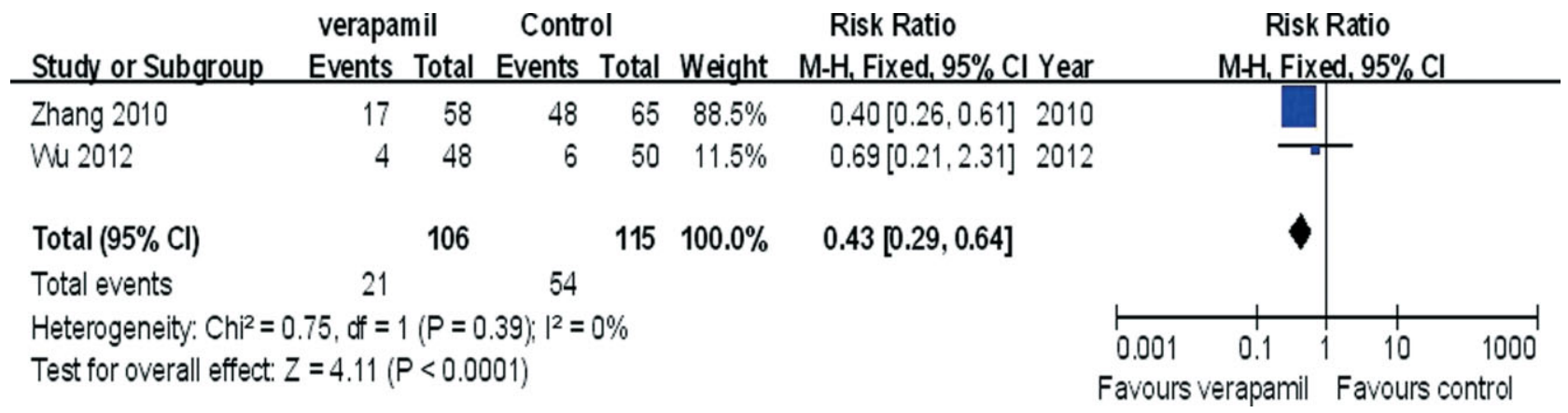

Figure 4. Pooled risk ratio of verapamil therapy vs control for TIMI myocardial perfusion grade (TMPG) after percutaneous coronary intervention. Abbreviations: $\mathrm{Cl}$, confidence interval; $\mathrm{M}-\mathrm{H}$, Mantel-Haenszel; TIMI, thrombolysis in myocardial infarction.

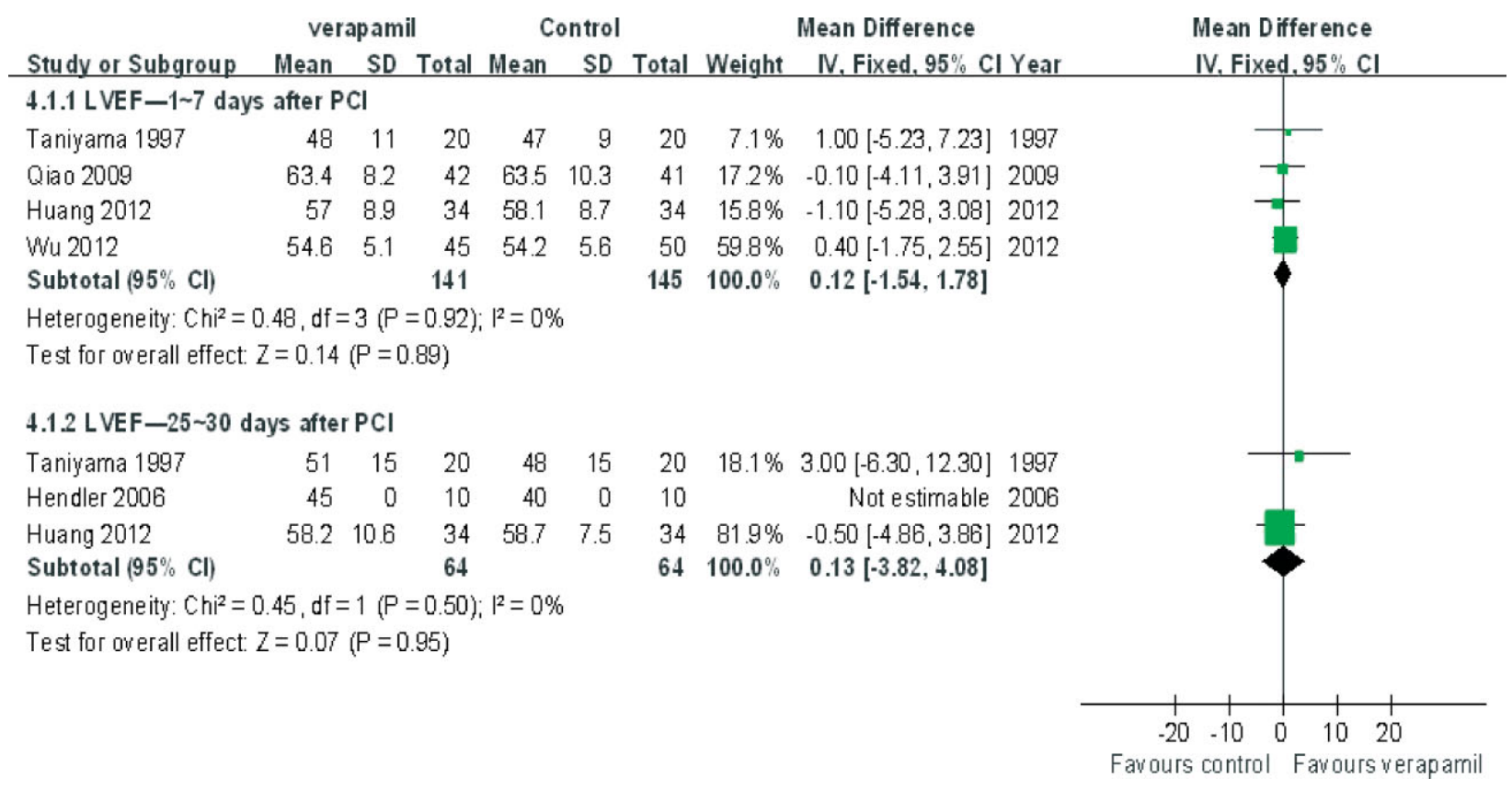

Figure 5. Pooled mean difference of verapamil therapy vs control for left ventricular ejection fraction (LVEF) after percutaneous coronary intervention (PCI). Abbreviations: $\mathrm{Cl}$, confidence intervals; IV, inverse variance; SD, standard deviation.

literature search we identified 28 items in PubMed, 81 in Embase, and 10 in the Cochrane database. After elaborative screening, we identified 7 randomized controlled trials (RCTs) that satisfied our inclusion criteria 7 studies. Those studies enrolled a total of 539 participants (266 for intracoronary verapamil injection and 273 as the control). Table 1 summarizes the study design and patient baseline characteristics for each of the eligible trials. No significant differences were found in baseline characteristics between the 2 groups. The mean follow-up period ranged from 1 to 6 months.

Of the 7 studies, patients in 2 studies $^{6,7}$ were randomized with no-reflow poststenting; in the other 5 studies, ${ }^{8-12}$ patients were randomized after PCI, no matter whether the no-reflow phenomenon happened or not. Patients in 6 studies $^{6,8,10-12}$ were preprocedurally treated with aspirin and clopidogrel, and only in 1 study $^{9}$ were patients given aspirin but without clopidogrel pre-PCI. Postintervention antiplatelet therapy consisted of aspirin and thienopyridines if stents were planted. The use of glycoprotein IIb/IIIa was unknown in the included studies. As to the complications related to verapamil, data were available in only 1 study, ${ }^{10}$ in which 9 patients developed transient heart block and were associated with a drop in systolic blood pressure, but none of this was life threatening. After 12 hour monitoring, all of the patients returned to normal. Of the 7 studies, infusion of verapamil was done through a microcatheter in 3 studies, ${ }^{6,11,12}$ a guiding catheter was used in 1 study, ${ }^{7}$ and 3 studies did not mention this item.

A summary of the methodological quality of the included studies was performed. However, only $3 \mathrm{RCT}^{6,11,12}$ reported the specific random methods that were used. In 1 study a block random approach was used, ${ }^{6}$ and in 2 other studies a random number table was used. ${ }^{11,12}$ None of the studies described the allocation concealments in detail. In 4 studies, blinded methods were used., $, 8,11,12$ Of those, 2 studies used a single-blind approach ${ }^{6,8}$ and 2 used a double-blind approach. ${ }^{11,12}$ Detailed methods used in these studies are presented in Table 2. 


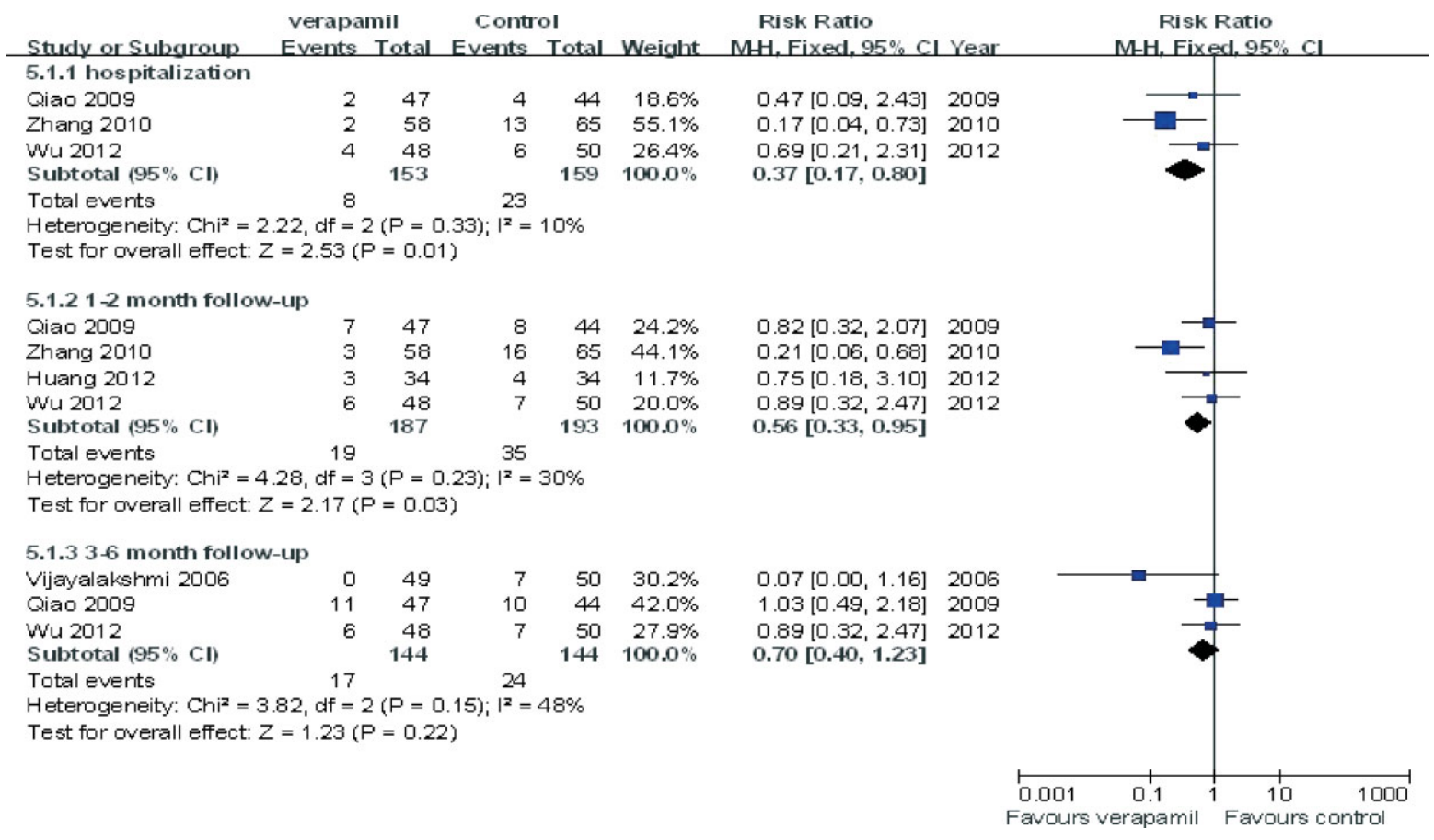

Figure 6. Pooled risk ratio of verapamil therapy vs control for major adverse cardiac events after percutaneous coronary intervention. Abbreviations: $\mathrm{Cl}$, confidence interval; M-H, Mantel-Haenszel.

The pooling analysis showed that intracoronary verapamil injection significantly decreased the occurrence of the coronary no-reflow phenomenon (RR: 0.33 , 95\% CI: 0.2 to $0.50 ; I^{2}=11 \%$; Figure 2) as well as reduced CTFC (WMD: -11.62 ; $95 \% \mathrm{CI}$ : -16.04 to $-7.21 ; I^{2}=0 \%$ ) (Figure 3) and improved TIMI myocardial perfusion grade (RR: 0.43; $95 \%$ CI: 0.29 to $0.64 ; I^{2}=0 \%$; Figure 4 ) in patients with ACS. Moreover, verapamil treatment showed superiority in reducing WMI compared to the control at day 1 and day 30 post-PCI (both $P<0.05$ ). However, administration of verapamil did not provide an additional improvement of LVEF regardless of the time that had past postprocedure (at 7 days, WMD: $-0.12 ; 95 \% \mathrm{CI}:-1.54$ to $1.78 ; I^{2}=0 \%$; at 25-30 days, WMD: -0.13 ; $95 \%$ CI: -3.82 to $4.08 ; I^{2}=0 \%$; Figure 5). Nevertheless, intracoronary verapamil injection was associated with a reduced risk of MACEs in the hospital (RR: 0.37 ; $95 \%$ CI: 0.17 to $0.80 ; I^{2}=10 \%$ ) and month 1 to 2 post-PCI (RR: 0.56 ; $95 \%$ CI: 0.33 to $0.95 ; I^{2}=30 \%$ ). Of note, the beneficial effect became nonsignificant at 3 to 6 months postprocedure (RR: $0.70 ; 95 \%$ CI: 0.40 to $1.23 ; I^{2}=48 \%$; Figure 6).

For all of the above analyses, sensitivity analyses yielded consistent results. Based on a visual inspection of the funnel plots, there was no evidence of publication bias for the included studies that assessed all clinical end points (Figure 7).

\section{Discussion}

The present study revealed that intracoronary verapamil injection is associated with a reduced incidence of coronary no-reflow and short-term MACEs in patients with ACS undergoing PCI. In addition, the procedure significantly

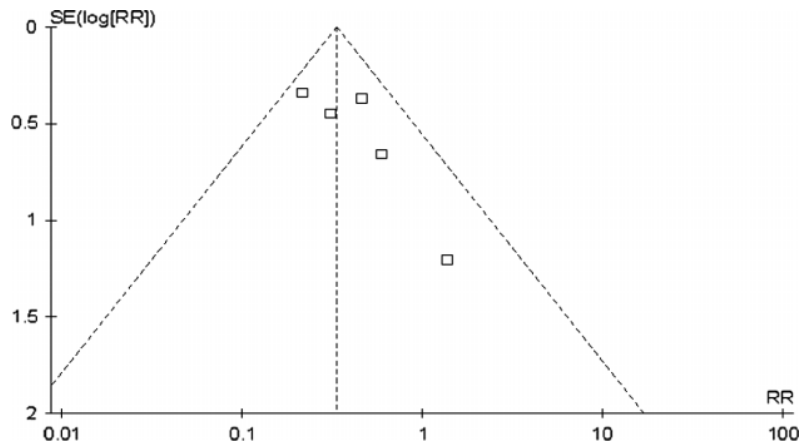

Figure 7. Funnel plot assessing publication bias of the included studies. Abbreviations: RR, risk ratio; SE, standard error.

reduced WMI compared to the control. However, verapamil treatment did not have a beneficial effect for improving cardiac systolic function.

As an L-type calcium channel blocker, verapamil improves microvascular dysfunction by releasing or preventing microvessel spasm and regulating its endothelial function ${ }^{13}$ The current study found a benefit of verapamil in preventing and treating coronary no-reflow phenomenon associated with PCI in patients with ACS. The drug significantly improved the TIMI grade with coronary no-reflow and reduced the incidence of PCI complications. Although administration of verapamil did not significantly improve LVEF 6 months post-PCI compared to the control, the treatment did reduce myocardial ischemia and decrease WMI at 30 days post-PCI. Moreover, verapamil also reduced the occurrence of MACEs in the hospital and 2 months postprocedure. 
Coronary no-reflow that is attributed to microcirculation dysfunction is the result of multiple factors. ${ }^{14}$ Since no-reflow may lead to the fatal complications and a poor clinical prognosis, it is critically important to prevent and treat PCI-related complications and improve the efficacy and safety of the procedure. Contemporary therapy of coronary no-reflow includes vasodilators as well as antithrombotic or thrombolytic drugs. ${ }^{15-17}$ Adenosine is regarded as an effective drug for reducing the incidence of no-reflow; however, it can increase the risk of heart block, hypotension, and other adverse reactions. Moreover, the half-life is relatively short, and thus the agent has to be administrated repeatedly. ${ }^{18}$ In addition, nitroprusside effectively improves coronary blood flow and prevents the occurrence of no-reflow, but the vasodilator can cause dose-related hypotension, which limits its clinical utility. ${ }^{19,20}$ In contrast to these agents, verapamil not only prevents the occurrence of coronary noreflow but also improves the short-term clinical prognosis. However, additional large-scale clinical studies are needed to further assess the utility of the drug and long-term outcomes. Nevertheless, verapamil may be a more promising adjuvant drug therapy that other agents currently available.

Some limitations of the present study deserve special consideration. First, the follow-up period of the 7 assessed trials was no more than 6 months. We cannot exclude that significant variances in clinical outcomes may emerge after a longer follow-up period. Second, due to the limited study number and population size, the power of the analysis might be restricted. Third, the retrospective nature of the study is also a limitation. Finally, The complications related to verapamil were only available in 1 study, ${ }^{10}$ in which 9 patients developed transient heart block that was associated with a drop in systolic blood pressure, but none of this was life threatening, and after being monitored for 12 hours all of the patients returned to normal. Therefore, these findings should be interpreted with caution and may warrant further investigation. Several clinical studies have shown that intracoronary nitroglycerin or heparinized saline injection have no impact on the improvement of coronary no-reflow. ${ }^{4,21}$ Based on these findings, we did not conduct further subgroup analyses for the control. Nevertheless, the sensitivity analyses and analysis of publication bias confirmed the overall results in the meta-analysis.

\section{Conclusion}

Taken together, intracoronary verapamil injection was found to be associated with an increased coronary TIMI grade in ACS patients undergoing PCI concomitant with coronary no-reflow. Moreover, the procedure significantly decreased ventricular motion capacity and reduced the incidence of MACEs. Larger-scale prospective randomized trials with a longer follow-up duration are required to further assess the efficacy and safety in patients with ACS as well as patients across the entire range of coronary artery diseases.

\section{References}

1. Dong-bao L, Qi H, Zhi L, et al. Predictors and long-term prognosis of angiographic slow/no-reflow phenomenon during emergency percutaneous coronary intervention for ST-elevated acute myocardial infarction. Clin Cardiol. 2010;33:E7-E12.

2. Chen Y, Yang Y. No-reflow phenomenon during percutaneous coronary intervention. Adv Cardiovasc Dis. 2005;26:5.

3. Porto I, Ashar V, Mitchell AR. Pharmacological management of no-reflow during percutaneous coronary intervention. Curr Vasc Pharmacol. 2006;4:95-100.

4. Werner GS, Lang K, Kunhnert H, et al. Intracoronary verapamil for reversal of no-reflow during coronary angioplasty for acute myocardial infarction. Catheter Cardiovasc Interv. 2002;57:444-451.

5. Higgins J, Green S, eds. Cochrane Handbook for Systematic Reviews of Interventions. Oxford, UK: Cochrane Collaboration; 2008.

6. Huang D, Qian J, Ge L, et al. Restoration of coronary flow in patients with no-reflow after primary coronary intervention of acute myocardial infarction (RECOVER). Am Heart J. 2012;164:394-401.

7. Zhang H, Cao Y, Wu Z, et al. Effect of intracoronary verapamil on no-reflow during percutaneous coronary intervention. Shangdong Med J. 2010;50:23.

8. Hendler A, Aronovich A, Kaluski E, et al. Optimization of myocardial perfusion after primary coronary angioplasty following an acute myocardial infarction. Beyond TIMI 3 flow. I Invasive Cardiol. 2006;18:32-36.

9. Taniyama $\mathrm{Y}$, Ito $\mathrm{H}$, Iwakura $\mathrm{K}$, et al. Beneficial effect of intracoronary verapamil on microvascular and myocardial salvage in patients with acute myocardial infarction. J Am Coll Cardiol. 1997;30:1193-1199.

10. Vijayalakshmi K, Whittaker VJ, Kunadian B, et al. Prospective, randomised, controlled trial to study the effect of intracoronary injection of verapamil and adenosine on coronary blood flow during percutaneous coronary intervention in patients with acute coronary syndromes. Heart. 2006;92:1278-1284.

11. Qiao Z, Pu J, Ding S, et al. Effect of intracoronary verapamil on coronary flow, myocardial perfusion and clinical outcome during percutaneous coronary intervention for acute myocardial infarction. Chin J Intervent Cardiol. 2009;17:6.

12. Wu M, Ye Z. Clinical efficacy and safety of intracoronary verapamil during percutaneous coronary intervention in patients with acute coronary syndromes. Chin J Geriatr. 2012;31:4.

13. Hang CL, Wang CP, Yip HK, et al. Early administration of intracoronary verapamil improves myocardial perfusion during percutaneous coronary interventions for acute myocardial infarction. Chest. 2005;128:2593-2598.

14. Movahed MR, Butman SM. The pathogenesis and treatment of no-flow occurring during percutaneous coronary intervention. Cardiovasc Revasc Med. 2008;9:56-61.

15. Micari A, Belcik TA, Balcells EA, et al. Improvement in microvascular reflow and reduction of infarct size with adenosine in patients undergoing primary coronary stenting. Am J Cardiol. 2005;96:1410-1415.

16. Forman MB, Jackson EK. Importance of tissue perfusion in ST segment elevation myocardial infarction patients undergoing reperfusion strategies: role of adenosine. Clin Cardiol. 2007;30:583-585.

17. de Lemos JA, Antman EM, Gibson CM, et al. Abciximab improves both epicardial flow and myocardial reperfusion in ST-elevation myocardial infarction. Observations from the TIMI 14 trial. Circulation. 2000;101:239-243.

18. Marzilli M, Orsini E, Marraccini P, et al. Beneficial effects of intracoronary adenosine as adjunct to primary angioplasty in acute myocardial infarction. Circulation. 2000;101: 2154-2159.

19. Barcin C, Denktas AE, Lennon RJ, et al. Comparison of combination therapy of adenosine and nitroprusside with adenosine alone in the treatment of angiographic no-reflow phenomenon. Catheter Cardiovasc Interv. 2004;61:484-491

20. Hillegass WB, Dean NA, Liao L, et al. Treatment of no-reflow and impaired flow with the nitric oxide donor nitroprusside following percutaneous coronary interventions: initial human clinical experience. J Am Coll Cardiol. 2001;37:1335-1343.

21. Kaplan BM, Benzuly KH, Kinn JW, et al. Treatment of no-reflow in degenerated saphenous vein graft interventions: comparison of intracoronary verapamil and nitroglycerin. Catheter Cardiovasc Diagn. 1996;39:113-118. 